\title{
Nutritional status among young adolescents attending primary school in Tanzania: contributions of mid-upper arm circumference (MUAC) for adolescent assessment
}

\author{
Margaret Lillie 1* $^{*}$, Isaac Lema ${ }^{2}$, Sylvia Kaaya ${ }^{2}$, Dori Steinberg ${ }^{1,3}$ and Joy Noel Baumgartner ${ }^{1}$
}

\begin{abstract}
Background: Adolescence is a critical time of development and nutritional status in adolescence influences both current and future adult health outcomes. However, data on adolescent nutritional status is limited in low-resource settings. Mid-upper arm circumference (MUAC) has the potential to offer a simple, low-resource alternative or supplement to body mass index (BMI) in assessing nutrition in adolescent populations.

Methods: This is secondary data analysis, from a cross-sectional pilot study, which analyses anthropometric data from a sample of young adolescents attending their last year of primary school in Pwani Region and Dar es Salaam Region, Tanzania ( $n=154 ; 92$ girls \& 62 boys; mean age 13.2 years).

Results: The majority of adolescents (75\%) were of normal nutritional status defined by BMI. Significantly more males were stunted than females, while significantly more females were overweight than males. Among those identified as outside the normal nutrition ranges, there was inconsistency between MUAC and BMI cut-offs. Bivariate analyses indicate that BMI and MUAC show a positive correlation for both female and male participants, and the relationship between BMI and MUAC was more strongly correlated among adolescent females.
\end{abstract}

Conclusions: Further studies are needed with more nutritionally and demographically diverse populations to better understand the nutritional status of adolescents and the practical contribution of MUAC cut-offs to measure adolescent nutrition.

Keywords: Tanzania, Mid-upper arm circumference, Adolescent nutrition, Assessment of nutritional status, Body mass index, Anthropometry

\section{Background}

Apart from reproductive health risk assessments, comprehensive adolescent health assessments are frequently neglected in clinical encounters, research, and programming in low and middle income countries (LMIC) [1, 2]. Without quality health information systems inclusive of adolescent health data, stakeholders are working in a data vacuum. This is particularly problematic for assessing adolescent nutritional status [3-5]. Historically,

\footnotetext{
* Correspondence: margaret.lillie@duke.edu

'Duke Global Health Institute, Duke University, Durham, USA

Full list of author information is available at the end of the article
}

undernutrition for children under age five has been the overwhelming nutritional priority in LMICs [6]. However, young adolescents in LMICs are also particularly at risk of undernutrition because they are often already stunted, have anaemia, and/or have existing infections from childhood [7]. For adolescent females, in particular, undernutrition contributes to a larger cycle of intergenerational undernutrition because adolescent mothers, and mothers who are undernourished from adolescence are more likely to have low birth weight infants which can lead to higher mortality rates, stunting, impaired mental development, and increase risk of chronic disease

(c) The Author(s). 2019 Open Access This article is distributed under the terms of the Creative Commons Attribution 4.0 International License (http://creativecommons.org/licenses/by/4.0/), which permits unrestricted use, distribution, and reproduction in any medium, provided you give appropriate credit to the original author(s) and the source, provide a link to the Creative Commons license, and indicate if changes were made. The Creative Commons Public Domain Dedication waiver (http://creativecommons.org/publicdomain/zero/1.0/) applies to the data made available in this article, unless otherwise stated. 
for their children [8-10]. While undernutrition continues to be a concern, with increasing globalization and development, LMICs are also experiencing an increase in overnutrition, creating a "dual burden" of under- and overnutrition [8].

Anthropometric measurements and their corresponding referent curves are positioned as important indicators for assessing nutritional status for adolescents due to their comparability across sex and age [11-13]. Age adjusted growth curves for body mass index (BMI) and height for children ages 5-19 were produced by the World Health Organization a decade ago [14]. In addition to height-for-age and BMI-for-age, mid-upper arm circumference (MUAC), or the measurement of the circumference of the arm halfway between the tip of the elbow and the tip of the shoulder, has the potential to offer a simple alternative or supplement to BMI in assessing nutrition in adolescent populations. The BMI measurement can be affected by factors that mask overall body mass, such as trunk oedema due to protein-energy malnutrition. MUAC is unaffected by this symptom [15]. BMI also requires adequate equipment, namely a standometer and functioning scale. In contrast, MUAC has the advantages of simple equipment (a standardized tape in millimetres created for MUAC measurements) and can be used by clinicians and nonclinicians alike in clinical and non-clinical settings, allowing for more efficiency in clinical practice and programs in low-resource settings [12, 16, 17].

Standardized cut-offs for MUAC in adolescent populations have not been established due to lack of studies and country level data on MUAC measurements in adolescents $[17,18]$. While there is an emerging body of evidence that MUAC and BMI correlate in adolescent populations, there are numerous calls for more evidence of this correlation for different age groups and geographic locations [17-23]. In support of broader use of MUAC, in 2017, Mramba et al. published MUAC-forage growth curves for 5-19year old children. These MUAC-for-age growth curves were created to be used with the WHO BMI-for-age growth curves for assessing undernutrition using the same data sets that were used to construct WHO reference curves for adolescents. These curves were validated using data from Kenya, Zimbabwe, and Uganda and deemed as effective as the WHO BMI-for-age cut-offs for assessing mortality risk due to undernutrition, however further research is needed to demonstrate the practical use of Mramba et al.'s findings [16, 24].

Understanding the nutritional status of adolescents is of particular importance in Tanzania. About one-quarter of the population of Tanzania are adolescents between 10 and 19 years old, yet the only national measures of adolescent nutrition are data from the 2015/2016 Demographic and Health Surveys (DHS) which reports anaemia and BMI measures for females 15 to 19 years old $[25,26]$. The DHS reports that of Tanzanian females ages 15-19, $47 \%$ are anaemic, $18 \%$ have a less than normal BMI, and $10 \%$ have a greater than normal BMI [26]. Although helpful measures, there is a distinct lack of information on the nutritional status of younger females ages 5-15 and males 5 years and above. This country-level data gap on nutritional status of young adolescents in Tanzania limits the country's ability to generate evidence for action to support programs that promote optimal health for adolescents during this critical period of growth [27].

While the Tanzania DHS does not broadly target adolescents, there are resources supported by the Tanzanian Ministry of Health, Community Development, Gender, Elderly and Children (MCDGC) that support nutrition assessment across the life course. The USAID supported Food and Nutrition Technical Assistance III Project (FANTA) and the MCDGC have partnered to not only create the Tanzania Food and Nutrition Center within the MCDGC but have also created the Tanzania Nutrition Assessment, Counseling, and Support (NACS) tools and trainings to assist providers in giving better nutrition care [27]. These tools support the WHO BMI-for-age zscores to be used for children and adolescents 5-17 years old and offer cut-offs for MUAC for adolescents 10-14 year olds and 15-17 year olds. While these resources undoubtedly support a range of nutrition programming efforts, there is no reported data-driven evidence base to support these cut-offs [15].

The primary aims of this secondary analysis were 1) to describe the nutritional status of in-school adolescents in two Standard 7 primary school classes in Tanzania using BMI, height, and MUAC, 2) to compare the currently available, age-specific MUAC cut-offs to BMI cut-offs, and 3) to determine the relationship between MUAC and BMI as indicators of nutritional status stratified by sex.

\section{Methods}

\section{Parent study/ethics}

Data from this study come from a pilot study assessing the feasibility and acceptability of Adolescent Wellness Visits, a service provided via a collaboration between schools and nearby health facilities, to provide reproductive health and non-reproductive health services including assessments and information on nutrition, vision, dental health, mental health, puberty, contraception, and HIV testing and counselling [Duke University Center for AIDS Research (CFAR) Small Grant, NIH 5P30 AI064518]. The intervention targeted Standard 7 students because they are in the last year of primary school before many students drop out prior to moving on to secondary school [26]. All study procedures were approved by the ethical review boards at Duke University, 
Muhimbili University of Health and Allied Sciences, and the National Institute of Medical Research of Tanzania. Before conducting the wellness visits, nurses at the study health facilities were trained and provided time to practice all aspects of the wellness visit. For anthropometric measurements, clinicians were trained by government district nutrition officers on how to measure height and weight and how to use MUAC measuring tapes and BMI wheels. Training participants practiced on the same person multiple times in order to achieve interrater reliability. Training participants were also provided with a manual to refer to after the training. For MUAC measurements, the manual contained information from the NACS job aid and training manual $[15,28]$.

\section{Participants}

All students currently enrolled in Standard 7 at the participating primary schools were eligible to participate in the study if they had given written assent themselves and had written consent from a parent/guardian. Of the 173 students in the two Standard 7 classes, 156 students were fully consented in the study and 154 completed the wellness visit.

\section{Setting}

Data were collected from a government primary school and a matched government health dispensary in Dar es Salaam Region (urban) and a government primary school and matched government district hospital in Pwani Region (peri-urban). These settings were chosen due to the district level interest in piloting the Adolescent Wellness Visit model.

\section{Data collection}

All participating adolescents were administered a survey after they completed their Adolescent Wellness Visit with the nurse at the health facility. The quantitative survey covered demographic characteristics and their experiences participating in the wellness visits. The assent and consent forms included permission for us to access their medical record data from the wellness visit encounter. Patient forms containing anthropometric measures were filled out by medical providers for each adolescent participant and entered electronically at the end of the survey by research assistants. Data was collected electronically on encrypted Android tablets through the Qualtrics Offline Surveys application Version 1.4 and uploaded to the Qualtrics server at the end of each day. Data collection instruments were translated into Kiswahili and administered by bilingual local research staff (recent MD graduates).

\section{Demographic measures}

We collected data on age, household size, religion, parent mortality, parent occupation, food security, puberty and socio-economic status. Food security was measured through a self-reported indicator of food access during the previous 4 weeks. The question is from the Household Food Insecurity Access Scale (In the past 4 weeks, did you ever worry that your household would not have enough food?) [29]. Pubertal status was measured through selfreported measures. Females were asked whether they had started their menstrual period. Males were asked if they have noticed any pubertal changes such as voice changes or experiencing a wet dream.

\section{Anthropometric measures}

Anthropometric measures were taken one time in a private room as part of the Adolescent Wellness Visit at the participating clinic. Height was measured in metres to the nearest centimetre with a clinic-provided stadiometer (light clothing, without shoes) as a continuous variable and transformed into height-for-age z-scores based on sex and age in months through the WHO STATA macro package based on the WHO height-forage curves [30]. Height-for-age z-scores were categorized based on the WHO stunting cut-offs (Stunted: <-2SD; Not stunted: $\geq-2 \mathrm{SD}$ ) [31]. Weight was measured in kilograms to the nearest tenth of a kilogram with a clinic-provided beam scale. BMI scores used in the study were calculated from the provider reported height and weight variables $(\mathrm{kg} / \mathrm{m} 2)$ and transformed into BMI-forage z-scores based on sex and age in months through the WHO STATA macro package based on the WHO BMI-for-age curves [31]. BMI-for-age z-scores were categorized based on the WHO BMI cut-offs for under and overnutrition (Obese: >2SD; Overweight: >1SD; Normal: 1 - -2SD; Thinness: <- 2 SD; Severe thinness: <- 3 SD) [32]. MUAC was measured in centimetres to the nearest millimetre as a continuous variable with standardized MUAC measuring tapes provided by the FANTA project. MUAC was measured on the bare arm of each participant halfway between the tip of the elbow and the tip of the shoulder, in accordance to the NACS job aid and manual. MUAC was transformed as a categorical variable using MUAC cut-offs for 10-14 years and 15 years and above recommended by NACS (Normal: $10-14$ years old: $\geq 18.5 \mathrm{~cm}, 15-17$ years old: $\geq 22.0 \mathrm{~cm}$; Moderate Acute Malnutrition: $10-14$ years old: $16.0-18.5 \mathrm{~cm}, 15-$ 17 years old: $18.5-22.0 \mathrm{~cm}$; Severe Acute Malnutrition: $10-14$ years old: $<16.0 \mathrm{~cm}, 15-17$ years old: $<18.5 \mathrm{~cm})$ $[15,28]$. MUAC was also transformed into a categorical variable using MUAC-for-age z-scores calculated and categorized using an $R$ Shiny web tool provided through correspondence with Jay Berkley and Lazarus Mramba, authors of the MUAC-for-age growth curves 
$(\mathrm{z}<-3,-2<\mathrm{z}>-3$, and $\mathrm{z}>-2)$ [24]. While not distinctly naming the categorized data, Mramba et al. found that children that had MUAC-for-age z-scores of -2 to -3 and less than -3 was associated with hazard ratios for death within 1 year compared to $\mathrm{z}$ scores of -2 or more [24].

\section{Data analysis}

For all continuous, bivariate analyses, a Wilcoxon rank sum test was used due to the non-parametric nature of the variables and the small sample size. For all categorical, bivariate analyses, a Fisher's Exact test was used for all variables that had cells with values less than 5 and a $x^{2}$ test was used for the remaining variables.

To determine the relationship between BMI calculations and MUAC measurements a Spearman's rank correlation coefficient was calculated due to the nonparametric distribution of MUAC values [33].

To examine the relationship between BMI and MUAC cut-offs, sensitivity and specificity were calculated for the NACS $(<18.5 \mathrm{~cm}$ for $10-14$ year olds; $<22.0 \mathrm{~cm}$ for 15-17 year olds) and Mramba et al. cut-offs $(\mathrm{z}<-2)$ when compared to BMI-for-age $\mathrm{z}$-score cut-offs $(\mathrm{z}<-$ $2)$. Here, sensitivity refers to the proportion of those who were undernourished based on age-specific" gold standard", who were also undernourished based on the comparison cut off. Specificity, in this case, refers to the proportion of those who were not undernourished (over-nourished or normal) based on the "gold standard", who were also not undernourished (normal) based on comparison cut-offs.

All analyses were conducted using the statistical software package STATA 15.

\section{Results}

\section{Demographics}

The socio-demographic characteristics of the study participants by study location are presented in Table 1 . There were 61 female participants and 41 male participants in Dar es Salaam and 31 female participants and 21 male participants in Pwani. Pwani participants were on average a year older than those in Dar es Salaam. Relatedly, more students in the Standard 7 class had reached puberty in Pwani, than in Dar es Salaam. More participants in Dar es Salaam reported having tap water inside their home and more participants in Pwani reported having electricity inside their homes. Father occupation differed between the two regions; with fathers in Pwani mainly working sales or services/clerical jobs and agriculture or fishing and fathers in Dar es Salaam mainly working in skilled and unskilled manual labour.

\section{Anthropometric measures}

Descriptive anthropometric measures categorized by sex are presented in Table 2 and summarized in the following sentences. Overall, females had statistically higher height, weight, BMI, and MUAC measurements than males. Although malnutrition indicated by cut-offs varied, up to $25 \%$ of the study population was outside of normal nutrition range. There were significant differences between males and females for height-for-age zscores measuring stunting, BMI-for-age z-scores measuring under- and overnutrition, and undernutrition using NACS guidelines cut-offs. There was no significant difference between self-reported puberty attainment between males and females. Significantly more males than females were stunted according to height-for-age z-score cut-offs. Males and females had a significantly different distribution of under- and overnutrition according to BMI-for-age $\mathrm{z}$-score cut-offs, with $17.4 \%$ of females being overweight compared to $4.8 \%$ of males and with 9.7\% of females being underweight compared to $17.7 \%$ of males. A greater proportion of males were categorized as having moderate acute malnutrition according to the NACS guidelines $(22.6 \%)$ than females (5.5\%). The same trend is presented using the Mramba et al. z-score cutoffs with $19.3 \%$ of males in the $<-2$ standard deviations category compared to $6.6 \%$ of females.

\section{Relationship between BMI and MUAC}

Bivariate analyses indicate that BMI and MUAC measurements show a positive correlation for both female and male participants, though the relationship between BMI and MUAC was more strongly correlated among adolescent females $(R=0.846, p=<0.001)$ compared to adolescent males $(R=0.459, p$-value $<0.001)$ (Fig. 1).

Exploratory sub-group analyses based on the BMI-forage cut-offs, performed to give an initial indication of direction and magnitude for future inquiry, revealed that in males and females who were undernourished, there was no correlation between BMI and MUAC (males: $n=11, r=0.442, p=0.173$; females: $n=9, r=0.246, p=$ 0.522 ). In females, there was a stronger correlation between BMI and MUAC in those who were overnourished ( $n=16, r=0.851, p=<0.001$; note: sample too small for boys, $n=3$ ). In males, the correlation between BMI and MUAC remained significant after categorizing between those who were stunted $(r=0.535, p=0.018)$ and those who were not stunted $(r=0.591, p<0.001)$. In females, there was no difference in correlation between BMI and MUAC between those who had reported attaining their menstrual cycle $(r=0.858, p<0.001)$ and those who had not $(r=0.803,=0.000$. For males, there was a weak correlation between BMI and MUAC in participants who had reported signs of puberty $(r=.5122$, $p=0.002)$ and there was no correlation between BMI 
Table 1 Socio-demographic characteristics of study sample $(n=154)$

\begin{tabular}{|c|c|c|c|c|}
\hline Variable & $\begin{array}{l}\text { Total } \\
n=154\end{array}$ & $\begin{array}{l}\text { Pwani } \\
n=52\end{array}$ & $\begin{array}{l}\text { Dar es Salaam } \\
n=102\end{array}$ & $p$-value \\
\hline Mean Age (range) & $13.2(11,18)$ & $13.8(12,16)$ & $12.9(11,18)$ & 0.000 \\
\hline \multirow[t]{2}{*}{ Mean \# of people (including self) in household (range) } & $6.4(3,25)$ & $6.8(3,25)$ & $6.3(3,15)$ & 0.596 \\
\hline & & $\%(n)$ & & \\
\hline Religion & & & & 0.000 \\
\hline Muslim & $63.6(98)$ & $92.3(48)$ & $49.0(50)$ & \\
\hline Christian & $35.7(55)$ & $5.8(3)$ & $51.0(52)$ & \\
\hline Father Muslim/ Mother Christian & $1(0.6)$ & $1(1.9)$ & $0(0.0)$ & \\
\hline Parent Mortality ${ }^{\mathrm{b}}$ & & & & 0.865 \\
\hline Both Parents Alive & $88.9(136)$ & $92.3(48)$ & $87.1(88)$ & \\
\hline Mother Deceased & $2.0(3)$ & $1.9(1)$ & $2.0(2)$ & \\
\hline Father Deceased & $8.5(13)$ & $5.8(3)$ & $9.9(10)$ & \\
\hline Both Parents Deceased & $0.7(1)$ & $0.0(0)$ & $1.0(1)$ & \\
\hline Mother's Occupation ${ }^{c}$ & & & & .083 \\
\hline Professional/Technical/Managerial & $2.0(3)$ & $0(0.0)$ & $3.1(3)$ & \\
\hline Clerical/ Sales \& Services & $30.9(46)$ & $41.2(21)$ & $25.5(25)$ & \\
\hline Manual (skilled or unskilled) & $28.9(43)$ & $17.7(9)$ & $34.7(34)$ & \\
\hline Agriculture/Fishing & $7.4(11)$ & $5.9(3)$ & $8.2(8)$ & \\
\hline Not currently working/Domestic Service & $26.9(40)$ & $33.3(17)$ & $23.5(23)$ & \\
\hline Do not know & $4.0(6)$ & $2.0(1)$ & $5.1(5)$ & \\
\hline Father's Occupation $^{d}$ & & & & 0.000 \\
\hline Professional/Technical/Managerial & $11.6(16)$ & $10.2(5)$ & $12.4(11)$ & \\
\hline Clerical/Sales \& Services & $13.8(19)$ & $24.5(12)$ & $8.9(7)$ & \\
\hline Manual (skilled or unskilled) & $45.7(63)$ & $16.3(8)$ & $61.8(55)$ & \\
\hline Agriculture/Fishing & $14.5(20)$ & $28.6(14)$ & $6.7(6)$ & \\
\hline Not currently working/Domestic Service & $2(1.5)$ & $0.0(0)$ & $2.3(2)$ & \\
\hline Do not know & $13.0(18)$ & $20.4(10)$ & $9.0(8)$ & \\
\hline Puberty & & & & 0.000 \\
\hline Started puberty & $52.6(81)$ & $67.3(35)$ & $45.1(46)$ & \\
\hline Not started puberty & $47.4(73)$ & $32.7(17)$ & $54.9(56)$ & \\
\hline Food Insecurity in Past 4 weeks & & & & 0.493 \\
\hline Yes & $11.0(17)$ & $9.6(5)$ & $14.7(15)$ & \\
\hline No & $89.0(137)$ & $90.4(47)$ & $85.3(87)$ & \\
\hline \multicolumn{5}{|l|}{ Equity Tool Items } \\
\hline \multicolumn{5}{|l|}{ Tap water inside home } \\
\hline Yes & $56.5(67)$ & $25.0(13)$ & $47.1(54)$ & 0.001 \\
\hline No & $43.5(87)$ & $75.0(39)$ & $52.9(48)$ & \\
\hline Toilet inside house or compound & & & & 0.274 \\
\hline Yes & $94.2(145)$ & $98.0(51)$ & $92.2(94)$ & \\
\hline No & $5.8(9)$ & $2.0(1)$ & $7.8(8)$ & \\
\hline Electricity inside house & & & & 0.006 \\
\hline Yes & $66.2(102)$ & $81.8(42)$ & $58.8(60)$ & \\
\hline No & $33.8(52)$ & $19.2(10)$ & $41.2(42)$ & \\
\hline
\end{tabular}


Table 1 Socio-demographic characteristics of study sample $(n=154)$ (Continued)

\begin{tabular}{|c|c|c|c|c|}
\hline Variable & $\begin{array}{l}\text { Total } \\
n=154\end{array}$ & $\begin{array}{l}\text { Pwani } \\
n=52\end{array}$ & $\begin{array}{l}\text { Dar es Salaam } \\
n=102\end{array}$ & $p$-value \\
\hline \multicolumn{5}{|c|}{ Someone in household with mobile phone } \\
\hline Yes & $100.0(154)$ & $100.0(52)$ & $100.0(102)$ & \\
\hline No & $0.0(0)$ & $0.0(0)$ & $0.0(0)$ & \\
\hline Television inside house & & & & 0.200 \\
\hline Yes & $66.2(102)$ & $73.1(38)$ & $62.7(64)$ & \\
\hline No & $33.8(52)$ & $26.9(14)$ & $37.3(38)$ & \\
\hline Main material of Floors & & & & 0.081 \\
\hline Earth, Sand, Dung & $7.1(11)$ & $9.6(5)$ & $5.9(6)$ & \\
\hline Ceramic Tiles & $24.0(37)$ & $13.5(7)$ & $29.4(30)$ & \\
\hline Cement & $68.2(105)$ & $76.9(40)$ & $63.7(65)$ & \\
\hline Carpet & $0.6(1)$ & $0.0(0)$ & $1.0(1)$ & \\
\hline
\end{tabular}

${ }^{a}$ No major differences in means and $p$-value with or without outlier

${ }^{\mathrm{b}} n=153^{\mathrm{c}} n=149^{\mathrm{d}} n=138$

Table 2 Anthropometric Measures and Pubertal Status by Sex

\begin{tabular}{|c|c|c|c|c|}
\hline Variable & $\begin{array}{l}\text { Total } \\
n=154\end{array}$ & $\begin{array}{l}\text { Males } \\
n=62\end{array}$ & $\begin{array}{l}\text { Females } \\
n=92\end{array}$ & $p$-value \\
\hline Mean Height (range) ${ }^{a}$ & $151.6 \mathrm{~cm}(130,170.9)$ & $149.5 \mathrm{~cm}(130,170.9)$ & $153.1(134.4,169.4)$ & 0.014 \\
\hline Mean Weight (range) & $42.2 \mathrm{~kg}(25,72)$ & $39.1 \mathrm{~kg}(26,58.9)$ & $44.2 \mathrm{~kg}(25,72)$ & 0.000 \\
\hline Mean BMI (range) & $18.3(12.8,31.2)$ & $17.4(13.5,30.3)$ & $18.8(12.8,31.2)$ & 0.002 \\
\hline \multirow[t]{2}{*}{ Mean MUAC (range) } & $22.3 \mathrm{~cm}(16.5,33)$ & $21.4 \mathrm{~cm}(18,33)$ & $23.0 \mathrm{~cm}(16.5,31.6)$ & 0.000 \\
\hline & & $\%(n)$ & & \\
\hline Pubertal Status & & & & 0.742 \\
\hline Started puberty & $52.6(81)$ & $54.8(34)$ & $51.1(47)$ & \\
\hline Not started puberty & $47.4(73)$ & $45.16(28)$ & $48.9(45)$ & \\
\hline Height-for-age z-score categorized (sex specific) & & & & 0.000 \\
\hline Not Stunted ( $\geq-2$ SD) & $85.7(132)$ & $69.3(43)$ & $96.7(89)$ & \\
\hline Stunted $(<-2$ SD) & $14.2(22)$ & $30.6(19)$ & $3.2(3)$ & \\
\hline BMI-for-age z-scores categorized (sex specific) & & & & 0.047 \\
\hline obese (>2SD) & $1.3(3)$ & $1.6(1)$ & $1.1(1)$ & \\
\hline overweight (> 1SD) & $11.0(17)$ & $3.2(2)$ & $16.3(15)$ & \\
\hline normal (1 - -2 SD) & $74.7(115)$ & $77.4(48)$ & $72.8(67)$ & \\
\hline thinness $(<-2$ SD) & $10.4(16)$ & $14.5(9)$ & $7.6(7)$ & \\
\hline severe thinness $(<-3 \mathrm{SD})$ & $2.6(4)$ & $3.2(2)$ & $2.1(2)$ & \\
\hline MUAC-for-age z-scores categorized (Mramba et al.) & & & & 0.004 \\
\hline$z>-2$ & $88.2(135)$ & $80.6(50)$ & $93.4(85)$ & \\
\hline$-2<z>-3$ & $10.5(16)$ & $19.3(12)$ & $4.4(4)$ & \\
\hline$z<-3$ & $1.31(2)$ & $0.0(0)$ & $2.2(0)$ & \\
\hline $\begin{array}{l}\text { MUAC categorized a,b (not delineated by sex, age adjusted) } \\
\text { Per NACS guidelines }\end{array}$ & & & & 0.002 \\
\hline Normal & $87.0(134)$ & $77.4(48)$ & $94.5(86)$ & \\
\hline Moderate acute malnutrition (MAM) & $12.3(19)$ & $22.6(14)$ & $5.5(5)$ & \\
\hline
\end{tabular}




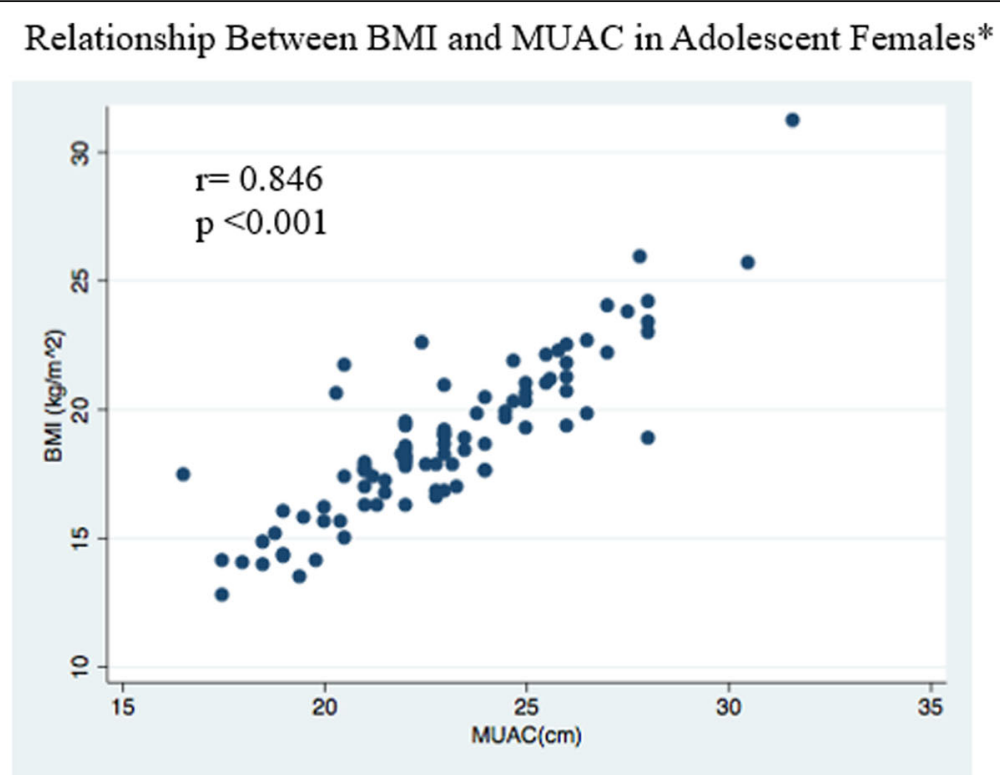

\section{Relationship Between BMI and MUAC in Adolescent Males*}

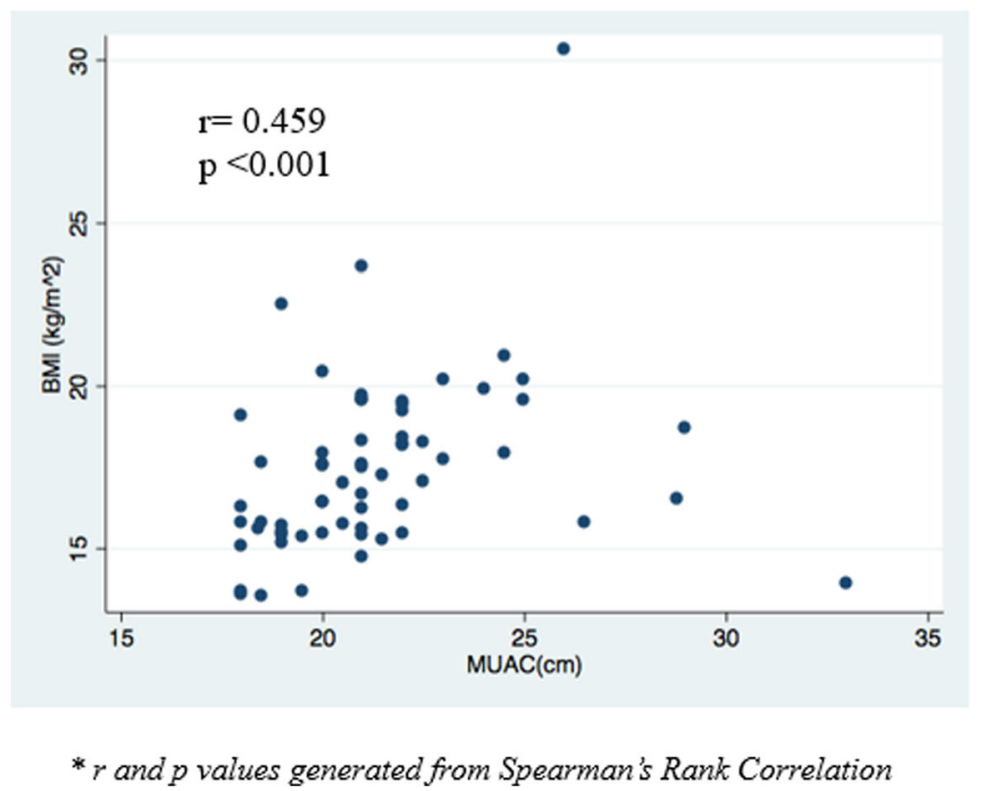

Fig. 1 Relationship between BMI and MUAC Measurements in Adolescent Females and Males

and MUAC for those who had not started puberty $(r=0.381, p=0.052)$.

\section{Comparing available MUAC cut-offs and BMI cut-offs}

Previous literature assessing the use of MUAC in adolescents has used BMI as a standard comparison $[17,18$, 20,34]. Sensitivity and specificity were calculated for the NACS and Mramba et al. cut-offs when compared to BMI-for-age z-score cut-offs. Both MUAC cut-offs had low sensitivity (NACS: $35.0 \%$; Mramba et al.:40.0\%) and high specificity (NACS: 90.1\%; Mramba: 92.5.3\%) (Fig. 2).
Additionally, if we are to compare the NACS MUAC cut-offs to the Mramba et al. cut-offs as a gold standard, NACS has $77 \%$ sensitivity and $96 \%$ specificity (Fig. 2).

\section{Discussion}

The data generated from the pilot study for Adolescent Wellness Visits offered an important opportunity to use secondary data to address the data gap in descriptive information about adolescent nutrition in Tanzania and to add to the continued inquiry of using MUAC in adolescent populations. Studies solely created to assess 


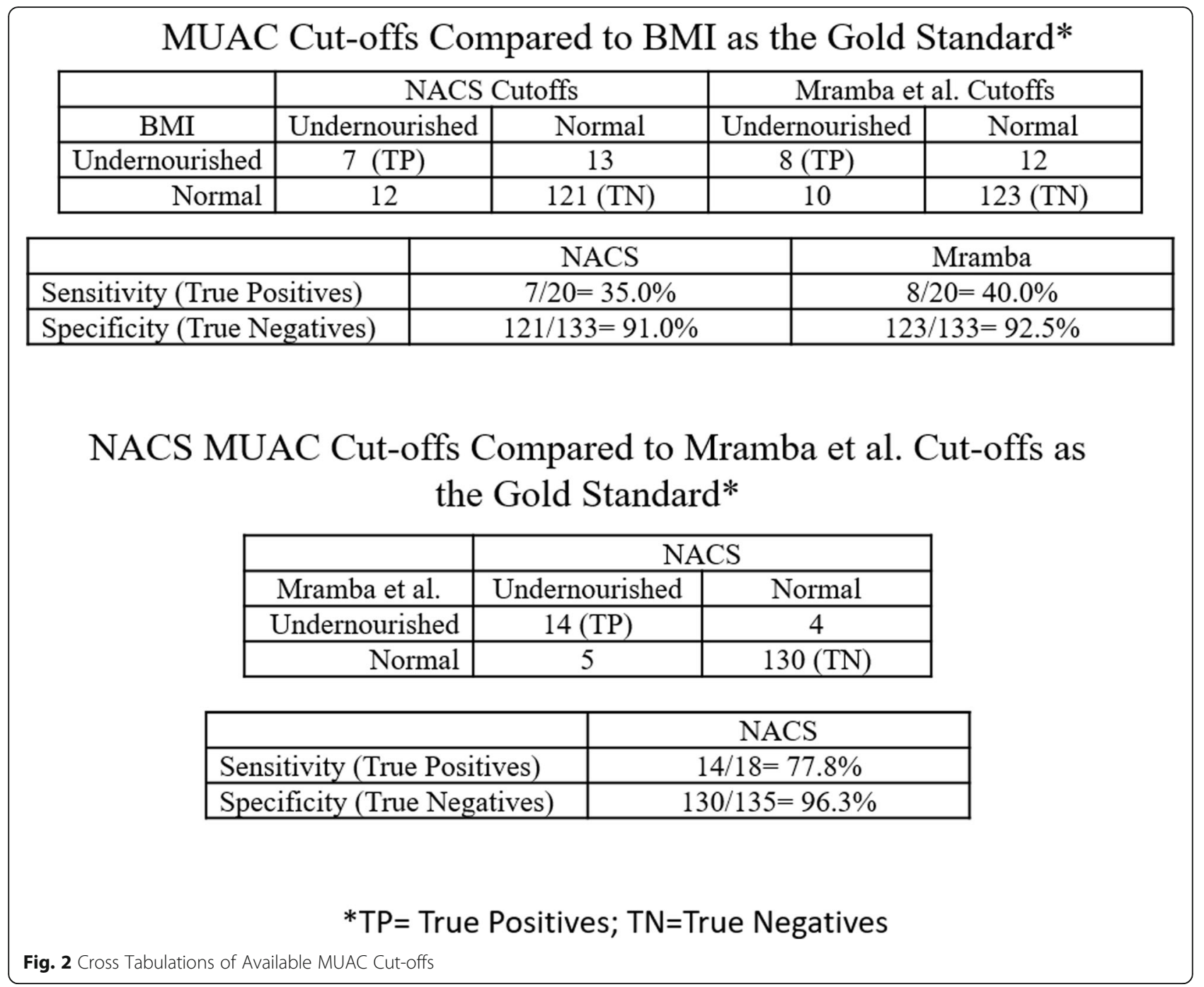

nutritional status, studies with larger sample sizes, and more population-level data collection are needed to confirm our findings and to make country level decisions about programing and policies concerning adolescent nutrition and MUAC cut-offs.

\section{Nutritional status of young school-age adolescents in Tanzania}

About one-quarter of our study participants were outside normal nutritional parameters based on BMI. Our adolescent study population was both under- and overnourished, while this result may not be generalizable to the Tanzanian adolescent population it offers an opportunity to consider how interventions and programming could dually address under- and overnutrition among adolescents.. Results also indicate that adolescent males and females may have different and distinct nutritional needs. We found that among this sample of Tanzanian adolescents there was significantly more stunting in males than in females. Stunting offers important information of the health status of the population considering that stunting is the result of, in part, poor maternal health and chronic infection $[8,9]$. Interpretation of the ten-fold difference in stunting status should be tempered by acknowledging the sample size and the need for more population based data from adolescent populations to confirm this finding. That said, this sex difference clearly aligns with the literature. A systematic review by Wamani et al., of DHS data from 10 sub-Saharan African countries, found that male children under-five have consistently higher rates of stunting than their female counterparts when considering both mean height-for age z-score and stunting prevalence [35]. Male participants in our sample also had higher rates of undernutrition. This aligned with trends showing differences in undernutrition between males and females in East Africa with more males ages 15-19 being underweight than their female counterparts (Uganda: $12.6 \%$ females, 26.3\% males; Rwanda: 10.9\% 
females, $30 \%$ males) [36, 37]. Both higher susceptibility to stunting and undernutrition in males is further confirmed in a systematic review by Akombi et al., determining the factors associated with stunting, wasting and underweight in Sub-Saharan Africa, which found that most studies in their review reported higher susceptibility to stunting, wasting, and underweight in male children than female children [38]. The results also highlight that the young adolescent females in our sample had higher rates of overnutrition than males. This follows the same trend as reported in other recent DHS data in East Africa with females ages 15-19 having higher prevalence of overweight or obesity than males ages 15-19 (Uganda: $11 \%$ females, $1 \%$ males; Rwanda: $13 \%$ females, $1 \%$ males) [36, 37]. While the Tanzania DHS does not report nutritional status for men, the percentage of women ages 15-19 in Tanzania who are overweight or obese has increased from $11 \%$ in the 1991-1992 survey to $28 \%$ in the 2015-2016 survey [26]. With clear data of under- and overnutrition in middle and later adolescences (ages 15-19), plus indications of both under- and overnutrition during younger adolescence ( ages 12-15), there is a distinct need for prevention efforts to start as early as possible to circumvent poor nutritional status continuing into adulthood. Adolescent specific nutrition programs could offer necessary sources of positive mental and physical growth during an important time in development especially when adolescents are at higher risk for adverse health outcomes due to stunting, undernutrition, and overnutrition [9].

\section{Relationship between MUAC and BMI}

Aligning with previous literature, the results of this study indicate that BMI and MUAC measurements are positively correlated for young adolescents [17-23]. Our finding are the first to compare the BMI and MUAC relationships stratified by sex and to show that the correlation between BMI and MUAC is weaker in males. Further studies replicating this finding are needed to better understand the cause of the weaker correlation and if this is due to random chance, true differences between males and females, or to systematic error such as outliers of BMI and MUAC in the male study sample, that boys are misclassified due to stunting, or due to measurement errors $[39,40]$.

Because MUAC and BMI show a stronger relationship in females who are overnourished than those who are undernourished, this indicates a need to better understand the use of MUAC in populations susceptible to overnutrition. There are some studies with evidence to support the use of MUAC to identify overweight and obese populations of adolescents [21, 41, 42], so it will be important to further consider the relationship between MUAC and BMI in future research to understand whether it is feasible to include cut-offs for overweight in existing MUAC cut-offs.
Based on our exploratory sub-group analysis of comparing BMI and MUAC by self-reported pubertal status in males and females, there is no evidence from the current study to suggest that pubertal status affects the $\mathrm{BMI}$ and MUAC relationship. Previous studies assessing the BMI and MUAC relationship in adolescents have not stratified the analysis by pubertal status $[17,18,20,21]$. Body proportions and fat distributions change rapidly during puberty; males gain more muscle mass in their trunk and upper body, while females gain more fat mass in their hips and thighs [43]. While on a population level, the effects of puberty may be normalized through age and sex standardized z-scores, it will be important to further consider how puberty may affect screening of individual children. In LMICs it will be prudent to understand whether BMI or MUAC is more or less valid when measuring the nutritional status of an adolescent pre- or post-puberty and whether it is more or less valid for boy or girls at different stages in puberty.

\section{Currently available MUAC and BMI cut-offs}

While we identified adolescents outside the normal nutritional ranges, the sensitivity and specificity results showed that there was inconsistency between the different cut-offs (WHO's BMI-for-age z-score cut-offs, cut-offs provided by the NACS materials, and Mramba et al.'s newly forms MUAC-for-age zscore cut-offs). Because the sensitivity of both MUAC cut-offs was low compared to BMI for age cut offs, we must consider what this means for the practical use of these measurements. For example, when using BMI and MUAC concurrently, which cut-off should prompt a referral? Because the NACS and the Mramba et al. cut-offs do not align, it will be important to also further consider the practical use of the Mramba et al. z-scores and how they can inform country-level MUAC cut-offs like the ones found in the NACS job aids and training manuals. For example, the Mramba et al. cut-offs could help inform sex-specific cut-offs for future NACS tools which currently do not have sex-specific cut-offs. The potential value of the MUAC measurement is limited to undernutrition because current cut-offs only assess undernutrition and not overnutrition. The available MUAC cut-offs only categorize those who are undernourished and do not provide cut-offs for those who are over nourished, representing a distinct complementary need for BMI as a common nutritional indicator and, again, a better understanding of MUAC and BMI in adolescents who are in non-normal categories of nutritional cut-offs. However, in fragile or vulnerable settings where high undernutrition is likely, the MUAC could be valuable tool for quick adolescent assessments. 


\section{Study strengths and limitations}

This was the first analysis to examine the relationship between BMI and MUAC in adolescents in Tanzania, a setting with limited information on young adolescent nutrition. Most nutrition information about adolescents in LMICs is taken at the clinic level, often in populations with HIV $[4,5]$. This study is unique in that it was collected in collaboration between schools and clinics among students of varying health and nutritional status. This study also had several limitations. First, due to the small sample size and the narrow scope of this study to two schools in Tanzania, generalizability among adolescents across Tanzania is limited. Second, while BMI is often used as the standard comparison to MUAC, this was the first time the participating clinicians were calculating BMI with BMI wheels and measuring MUAC on adolescents. Height, weight, and MUAC are all measurements that are prone to measurement error due to inter-observer differences in measurement or rounding errors [44]. Additionally, since this was a pilot study using secondary data and given the limitations of our measures of puberty, in future studies it will be important include more robust measures of puberty in population-based nutrition data to better understand the effect of puberty on the BMI and MUAC relationship. Alternative indicators for puberty may include multiple self-reported questions about various stages of puberty or a physical exam by a clinician [44].

\section{Research recommendations}

Future population-based studies need to be done with more nutritionally and socially diverse populations in Tanzania and globally, to be able to better understand the nutritional status of adolescents and to contribute data for cut-off recommendations. For example, by adding children and adolescents ages 5-15 in a subsample of the DHS with indicators like BMI and MUAC, Tanzania and other LMICs will be better equipped to track and understand the nutritional status of their adolescents. Larger and more diverse sample sizes will contribute to informing sex-specific cut-offs for MUAC in further research. The relative contribution of using the MUAC as an indicator of overnutrition requires additional research as does how stunting affects under and overnutrition measurements.

\section{Program and policy recommendations}

The high prevalence of stunting in this population of adolescent males indicates a distinct need to target cycles of undernutrition and chronic infection that contribute to stunting. This requires continuing to target mothers and children under five, but it also requires extending the age range of "child" services so that children above 5 years old also receive necessary and affordable nutrition support services. During a study dissemination meeting with Tanzanian stakeholders about the larger study of wellness visits held in October 2018, parents and school staff were acutely aware of the nutritional issues for young adolescents and were eager to seek information and guidance from health providers about how to improve the nutritional status of adolescents. This awareness was articulated with an acknowledgement that nutritional status and educational outcomes are closely linked and both the health and education sectors can work together to support this agenda.

More pragmatically, within health services, it would still be optimal for both the MUAC and BMI measures to be taken for adolescent assessments particularly because as indicated in global guidelines, the MUAC is accurate in cases where BMI is not a proper measurement due to conditions affecting one's weight such as adolescents who are pregnant or have HIV. In addition, in the absence of the equipment or training to properly calculate BMI, the MUAC could offer a feasible and informative alternative although additional research on adolescent cut-offs would strengthen this recommendation.

\section{Conclusion}

This study contributes to the literature on adolescent nutrition in a low-resource setting and to the evidence base on MUAC assessments as an indicator for nutritional status in adolescents. Given that BMI and MUAC are correlated in this population of adolescents, MUAC may offer a promising method for simple, low resource nutrition screening when BMI is not possible. More studies are needed in adolescents who are identified as having nonnormal nutritional status to understand the relationship between BMI and MUAC on a more nuanced level.

\section{Abbreviations \\ BMI: Body Mass index; DHS: Demographic and Health Service; LMIC: Low and Middle Income Countries; MUAC: Mid-Upper Arm Circumference; \\ NACS: Tanzania Nutrition Assessment, Counseling, and Support \\ Acknowledgements \\ We thank our research assistants Helga Herman, Paul Mwasapi, Elly Josaphat, and Alice Kyara. We would also like to thank all of the stakeholders who made this study possible including students, parents, and those from the education and health sectors. Thank you to Lazarus Mramba and Jay Berkley for providing us with the R-Shiny application to calculate age-specific MUAC z-scores.}

\section{Authors' contributions}

ML wrote the manuscript with support from IL, SK, DS and JNB. JNB and DS advised ML for her masters' thesis for which this manuscript is based and contributed to the interpretation of the analyses. IL and SK contributed to the design and implementation of the research. All authors (ML, IL, SK, DS, JNB) revised drafts and approved the final manuscript.

\section{Funding}

Small Grant from the Duke University Center for AIDS Research (CFAR), an $\mathrm{NIH}$ funded program (5P30 Al064518); PI: Baumgartner JN. The funding body had no part in design, data collection, analysis, interpretation of data, or manuscript writing. 


\section{Availability of data and materials}

The datasets generated and/or analyzed during the current study are not publicly available but are available from the corresponding author on reasonable request.

\section{Ethics approval and consent to participate}

All study procedures were approved by the ethical review boards at Duke University, Muhimbili University of Health and Allied Sciences, and the National Institute of Medical Research of Tanzania. All participants provided written assent and written consent was obtained from their parents.

\section{Consent for publication}

Not Applicable.

\section{Competing interests}

The authors declare that they have no competing interests.

\section{Author details}

'Duke Global Health Institute, Duke University, Durham, USA. ${ }^{2}$ School of Medicine, Muhimbili University of Health \& Allied Sciences (MUHAS), Dar es Salaam, Tanzania. ${ }^{3}$ School of Nursing, Duke University, Durham, USA.

Received: 8 July 2019 Accepted: 5 November 2019

Published online: 27 November 2019

\section{References}

1. Mehrotra A, Zaslavsky AM, Ayanian JZ. Preventive health examinations and preventive gynecological examinations in the united states. Arch Intern Med. 2007;167(17):1876-83

2. Patton GC, Sawyer SM, Santelli JS, Ross DA, Afifi R, Allen NB, et al. Our future: a Lancet commission on adolescent health and wellbeing. Lancet. 2016;387(10036):2423-78.

3. Delisle H, Chandra-Mouli V, de Benoist B. Should Adolescents Be Specifically Targeted for Nutrition in Developing Countries? To Address Which Problems, and How? Geneva: WHO; 2005.

4. Lassi ZS, Mansoor T, Salam RA, Bhutta SZ, Das JK, Bhutta ZA. Review of nutrition guidelines relevant for adolescents in low- and middle-income countries. Ann N Y Acad Sci. 2017;1393(1):51-60.

5. Khara T, Mates E. Adolescent Nutrition: Policy and Programming in SUN+ Countries. London: Save the Children; 2015.

6. Prendergast AJ, Humphrey JH. The stunting syndrome in developing countries. Paediatri Int Child Health. 2014;34(4):250-65.

7. Thurnham DI. Nutrition of Adolescent Girls in Low and Middle Income Countries. 2013;27(3).

8. West KP, Stewart CP, Caballero B, Black RE. Chapter 6: nutrition. In: Merson $\mathrm{MH}$, Black RE, Mills AJ, editors. Global Health: diseases, programs, systems, and policies. 3rd Edition ed. Jones \& Bartlett Learning: Burlington; 2012.

9. Black RE, Victora CG, Walker SP, Bhutta ZA, Christian P, de Onis M, et al. Maternal and child undernutrition and overweight in low-income and middle-income countries. Lancet. 2013;382(9890):427-51.

10. Fourth Report on the World Nutrition Situation. Geneva: United Nations Administrative Committee on Coordination/ Sub Committee on Nutrition in collaboration with International Food Policy Research Institute; 2000.

11. Woodruff BA, Duffield A. Assessment of Nutritional Status of Adolescents in Emergency-Affected Populations. 2000 [Available from: www.ennonline.net/ adolescentnutritionassessment.

12. Cogill B. Guide to Anthropometry: A Practical Tool for Program Planners, Managers, and Implementers. Food and Nutrition Technical Assistance (FANTA). Washington, D.C.: Academy for Educational Development; 2003.

13. Wang Y, Chen $\mathrm{H}$-J. Use of percentiles and Z-scores in anthropometry. In: Preedy VR, editor. Handbook of anthropometry: physical measures of human form in health and disease. New York: Springer New York; 2012. p. 29-48.

14. de Onis M, Onyango AW, Borghi E, Siyam A, Nishida C, Siekmann J. Development of a WHO growth reference for school-aged children and adolescents. Bull World Health Organ. 2007;85(9):660-7.

15. Tanzania Food and Nutrition Centre (TFNC). Nutrition Assessment, Counselling and Support (NACS): Reference Manual for Training Health Facility-Based Service Providers. Dar es Salaam: TFNC; 2016.

16. Cashin K, Oot L. Guide to Anthropometry: A Practical Tool for Program Planners, Managers, and Implementers. Washington: Food and Nutrition Technical Assistance III Project (FANTA)/FHI 360; 2018.
17. Jeyakumar A, Ghugre P, Gadhave S. Mid-upper-arm circumference (MUAC) as a simple measure to assess the nutritional status of adolescent girls as compared with BMI. ICAN: Infant, Child, \& Adolescent Nutrition. 2013;5(1):22-25.

18. Tang AC, Dong K, Deitchler M, Chung M, Maalouf-Manasseh Z, Tumilowicz A, et al. Use of Cutoffs for Mid-Upper Arms Circumference (MUAC) as an Indicator or Predictor of Nutritional and Health-Related Outcomes in Adolscents and Adults: A Systematic Review. Washington, DC: FHI 360/FANTA; 2013.

19. Kulathinal S, Freese R, Korkalo L, Ismael C, Mutanen M. Mid-upper arm circumference is associated with biochemically determined nutritional status indicators among adolescent girls in Central Mozambique. Nutr Res. 2016;36(8):835-44.

20. Dasgupta A, Butt A, Saha TK, Basu G, Chattopadhyay A, Mukherjee A. Assessment of malnutrition among adolescents:can BMl be replaced by MUAC. Indian J Community Med. 2010;35(2):276-9.

21. Craig E, Bland R, Ndirangu J, Reilly JJ. Use of mid-upper arm circumference for determining overweight and overfatness in children and adolescents. Arch Dis Child. 2014;99(8):763-6.

22. Bahwere P. Anthropometric cut-off points for older children and adolescents in Syria. Field Exch. 2017;54:44.

23. Ayatollahi SMT, Bagheri Z, Heydari ST. Agreement analysis among measures of thinness and obesity assessment in Iranian school children and adolescents. Asian J Sports Med. 2013;4(4):272-80.

24. Mramba L, Ngari M, Mwangome M, Muchai L, Bauni E, Walker AS, et al. A growth reference for mid upper arm circumference for age among school age children and adolescents, and validation for mortality: growth curve construction and longitudinal cohort study. BMJ. 2017;358.

25. UNICEF. Adolescence in Tanzania Dar es Salaam, Tanzania: United Nations Chilren's Fund (UNICEF); 2011 [Available from: https://www.unicef.org/ infobycountry/files/TANZANIA_ADOLESCENT_REPORT_Final.pdf.

26. MoHCDGEC/MoH/NBS/OCGS/ICF. Tanzania Demographic and Health Survey and Malaria Indicator Survey 2015-2016. Dar es Salaam, Tanzania \& Rockville, Maryland, USA: Ministry of Health, Community Development, Gender, Elderly and Children [Tanzania Mainland], Ministry of Health (MoH) [Zanzibar], National Bureau of Statistics (NBS), Office of the Chief Government Statistician (OCGS), and ICF; 2016.

27. FANTA/USAID. Tanzania Nutrition Assessment, Counseling, and Support (NACS) Tools: FHI 360; 2013-2018 [Available from: https://www.fantaproject. org/tools/tanzania-nutrition-assessment-counseling-and-support-nacs-tools. Accessed 1 Jan 2017.

28. Tanzania Food and Nutri on Centre (TFNC). Nutrition Assessment, Counselling and Support (NACS): Job Aids for Health Facility-Based Service Providers. Dar es Salaam: TFNC; 2016.

29. Coates J, Swindale A, Bilinsky P. Household Food Insecurity Access Scale (HFIAS) for Measurement of Household Food Access: Indicator Guide, vol. 3. Washington: FHI 360/FANTA.; 2007.

30. WHO. Growth Reference Data for 5-19 Years: World Health Organization (WHO); 2007 [Available from: http://www.who.int/growthref/en/.

31. WHO. Height-for-age (5-19 years): World Health Organization (WHO); 2007 [Available from: http://www.who.int/growthref/who2007_height_for_age/en/.

32. WHO. BMI-for-age (5-19 years): World Health Organization (WHO); 2007 [Available from: http://www.who.int/growthref/who2007_bmi_for_age/en/.

33. Kirkwood BR, Stern JAC. Essential medical statistics. 2nd ed. Malden: Blackwell Publishing Company; 2003.

34. Reilly JJ. Mid-upper arm circumference (MUAC): new applications for an old measure. Arch Dis Child. 2017;102(1):1-2.

35. Wamani H, Astrom AN, Peterson S, Tumwine JK, Tylleskar T. Boys are more stunted than girls in sub-Saharan Africa: a meta-analysis of 16 demographic and health surveys. BMC Pediatr. 2007;7:17.

36. NISR/MOH/IFC. Rwanda Demographic and Health Survey 2014-15. Kigali, Rwanda \& Rockville, Maryland, USA: National Institute of Statistics of Rwanda, Ministry of Finance and Economic Planning/Rwanda, Ministry of Health/Rwanda, and ICF International; 2015.

37. UBOS/IFC. Uganda Demographic and Health Survey 2016. Kampala, Uganda \& Rockville, Manyland, USA: Uganda Bureau of Statistics and IFC International 2017.

38. Akombi BJ, Agho KE, Hall JJ, Wali N, Renzaho AMN, Merom D. Stunting, wasting and underweight in sub-Saharan Africa: a systematic review. Int J Environ Res Public Health. 2017;14(8):863.

39. Doak C. Is body mass index an appropriate proxy for body fat in children? 2013. p. 64-91.

40. Pongcharoen $T$, Judprasong $K$, Jitngarmkusol $S$, Kriengsinyos $W$, Winichagoon P. Body mass index is associated with fat mass in normal, 
overweight/obese, and stunted preschool children in Central Thailand. Asia Pac J Clin Nutr. 2017;26(4):686-91.

41. Chaput JP, Katzmarzyk PT, Barnes JD, Fogelholm M, Hu G, Kuriyan R, et al. Mid-upper arm circumference as a screening tool for identifying children with obesity: a 12-country study: Pediatric Obesity; 2016. n/a-n/a

42. Sanguansak Rerksuppaphol LR. Mid-upper-arm circumference and arm-toheight ratio to identify obesity in school-age children. Clin Med Res. 2017; 15(3-4):53-8.

43. Loomba-Albrecht LA, Styne DM. Effect of puberty on body composition. Curr Opin Endocrinol Diabetes Obes. 2009;16(1):10-5.

44. Dorn LD, Biro FM. Puberty and its measurement: a decade in review. J Res Adolesc. 2011;21(1):180-95.

\section{Publisher's Note}

Springer Nature remains neutral with regard to jurisdictional claims in published maps and institutional affiliations.

Ready to submit your research? Choose BMC and benefit from:

- fast, convenient online submission

- thorough peer review by experienced researchers in your field

- rapid publication on acceptance

- support for research data, including large and complex data types

- gold Open Access which fosters wider collaboration and increased citations

- maximum visibility for your research: over $100 \mathrm{M}$ website views per year

At BMC, research is always in progress.

Learn more biomedcentral.com/submissions 